\title{
Gang der Darstellung
}

Die Arbeit gliedert sich in insgesamt vier Kapitel. Aus methodischer Perspektive wird kapitelübergreifend ein Schwerpunkt auf die praktische Rechtsanwendung und die Erprobung aller im Verlaufe der Arbeit aufgeworfenen Fragen und möglichen Antworten hierzu in der konkreten Subsumtion anhand von Beispielsfällen gelegt.

Im ersten Kapitel werden schuldverhältnisbedingte Nebenpflichten einem Gegenwartsvergleich mit deliktischen Jedermannspflichten unterzogen. Dabei werden zunächst die begrifflichen Grundlagen des Themas einer Analyse unterzogen. Anschließend werden die Unterschiede zwischen der Haftung aus einem Schuldverhältnis und der Haftung aus Jedermannsrecht im sachlichen Kontext der Nebenpflichten beleuchtet. Dabei wird - mit Blick auf die von der Arbeit später aufgestellter These der teleologischen Subsidiarität von Nebenpflichten ${ }^{78}$ - insbesondere ein Augenmerk darauf gelegt, inwieweit die Praxis hinsichtlich Nebenpflichten eine vom Deliktsrecht unabhängige Prüfung vornimmt, in welchem dogmatischen Verhältnis schuldverhältnisbedingte Nebenpflichten zu Jedermannspflichten derzeit stehen und in welchem Ausmaß die Annahme von Nebenpflichten notwendig ist, um eine Haftung des Schädigers nach geltendem Recht zu ermöglichen.

Im zweiten Kapitel wird die Funktion von § 241 II BGB untersucht. Dabei wird zunächst die Begründungsbedürftigkeit von Nebenpflichten hergeleitet (Warum ist eine Begründung für die Annahme einer Nebenpflicht überhaupt erforderlich?). Anschließend wird der methodische Weg zur Begründung einer Nebenpflicht analysiert (Wie wird eine Nebenpflicht methodisch begründet?). Schließlich werden Haftungsgrund und Funktion von $\S 241$ II BGB durch Auslegung von $\S 241$ II BGB untersucht (Wie und mit Bezug auf welche Funktion wird eine Nebenpflicht dogmatisch begründet?). Der Schwerpunkt liegt dabei in der Herausarbeitung der telologischen Subsidiarität von Nebenpflichten, welche insbesondere gegenüber den Jedermannspflichten greift.

S.u., S. $270 \mathrm{ff}$. 
Im dritten Kapitel werden die Folgen der teleologischen Subsidiarität von Nebenpflichten in der Rechtsanwendung untersucht. Zunächst werden methodische Konsequenzen aus der vorher begründeten teleologischen Subsidiarität schuldverhältnisbedingter Nebenpflichten in Form eines eigenen, doppelfunktionalen Ansatzes hinsichtlich $\S 241$ II BGB gezogen (,Was folgt aus der teleologischen Subsidiarität von § 241 II BGB für die Prüfungsmethodik?“). Nach welchen Maßstäben Lücken durch die Annahme von Nebenpflichten in der Subsumtion zu schließen sind (,Was sind die inhaltlichen Maßstäbe für die Annahme von Nebenpflichten unter Berücksichtigung deren teleologischer Subsidiarität als Prämisse?"), wird für jeden der drei zentralen Haftungsvorteile des Schuldverhätnisrechts gegenüber dem Jedermannsrecht - erweiterter Vermögensschutz, strengeres Einstehen für das Verhalten von Drittpersonen sowie die Beweislastumkehr des § 280 I 2 BGB - separat untersucht. Dabei wird ein Schwerpunkt darauf gelegt, die Maßstäbe der ökonomischen Analyse in die praktische Rechtsanwendung zu integrieren.

Das vierte und letzte Kapitel dient der Zusammenführung aller bis dahin gesammelten Befunde. Die zentralen Ergebnisse der vorherigen Kapitel werden dabei in Thesengruppierungen gesammelt. Anschließend wird ein grobes Subsumtionsschema für die praktische Rechtsanwendung vorgeschlagen, welches anhand eines letzten Beispielsfalls erprobt wird. Zuletzt wird ein Resümee gezogen, welches die Ergebnisse der Arbeit im rechtswissenschaftlichen Diskurs verortet und weiteren Forschungsbedarf aufzeigt.

\footnotetext{
Open Access Dieses Kapitel wird unter der Creative Commons Namensnennung 4.0 International Lizenz (http://creativecommons.org/licenses/by/4.0/deed.de) veröffentlicht, welche die Nutzung, Vervielfältigung, Bearbeitung, Verbreitung und Wiedergabe in jeglichem Medium und Format erlaubt, sofern Sie den/die ursprünglichen Autor(en) und die Quelle ordnungsgemäß nennen, einen Link zur Creative Commons Lizenz beifügen und angeben, ob Änderungen vorgenommen wurden.

Die in diesem Kapitel enthaltenen Bilder und sonstiges Drittmaterial unterliegen ebenfalls der genannten Creative Commons Lizenz, sofern sich aus der Abbildungslegende nichts anderes ergibt. Sofern das betreffende Material nicht unter der genannten Creative Commons Lizenz steht und die betreffende Handlung nicht nach gesetzlichen Vorschriften erlaubt ist, ist für die oben aufgeführten Weiterverwendungen des Materials die Einwilligung des jeweiligen Rechteinhabers einzuholen.
}



